\title{
Time and amplitude calibration of the Baikal-GVD neutrino telescope
}

\author{
A.D. Avrorin ${ }^{a}$, A.V. Avrorin ${ }^{a}$, V.M. Aynutdinov ${ }^{a}$, R. Bannash ${ }^{g}$, I.A. Belolaptikov ${ }^{b}$, \\ D.Yu. Bogorodsky ${ }^{c}$, V.B. Brudanin ${ }^{b}$, N.M. Budnev ${ }^{c}$, I.A. Danilchenko ${ }^{a}$, G.V. \\ Domogatsky $^{a}$, A.A. Doroshenko ${ }^{a}$, A.N. Dyachok ${ }^{c}$, Zh.-A.M. Dzhilkibaev ${ }^{a}$, S.V. \\ Fialkovsky $^{e}$, A.R. Gafarov ${ }^{c}$, O.N. Gaponenko ${ }^{a}$, K.V. Golubkov ${ }^{a}$, T.I. Gress ${ }^{c}$, Z. Honz ${ }^{b}$, \\ K.G. Kebkal ${ }^{g}$, O.G. Kebkal ${ }^{g}$, K.V. Konischev ${ }^{b}$, A.V. Korobchenko ${ }^{b}$, A.P. Koshechkin ${ }^{a}$, \\ F.K. Koshel ${ }^{a}$, A.V. Kozhin ${ }^{d}$, V.F. Kulepov ${ }^{e}$, D.A. Kuleshov ${ }^{a}$, V.I. Ljashuk ${ }^{a}$, M.B. \\ Milenin $^{e}$, R.A. Mirgazov ${ }^{c}$, E.R. Osipova ${ }^{d}$, A.I. Panfilov ${ }^{a}$, L.V. Pan'kov ${ }^{c}$, E.N. \\ Pliskovsky $^{b}$, M.I. Rozanov ${ }^{f}$, E.V. Rjabov ${ }^{c}$, B.A. Shaybonov ${ }^{* b}$, A.A. Sheifler ${ }^{a}$, M.D. \\ Shelepov $^{a}$, A.V. Skurihin ${ }^{d}$, A.A. Smagina ${ }^{b}$, O.V. Suvorova ${ }^{a}$, V.A. Tabolenko ${ }^{c}$, B.A. \\ Tarashansky $^{c}$, S.A. Yakovlev ${ }^{g}$, A.V. Zagorodnikov ${ }^{c}$, V.A. Zhukov ${ }^{a}$, and V.L. Zurbanov ${ }^{c}$ \\ ${ }^{a}$ Institute for Nuclear Research, Moscow, 117312 Russia \\ ${ }^{b}$ Joint Institute for Nuclear Research, Dubna, 141980 Russia \\ ${ }^{c}$ Irkutsk State University, Irkutsk, 664003 Russia \\ ${ }^{d}$ Institute of Nuclear Physics, Moscow State University, Moscow, 119991 Russia \\ ${ }^{e}$ Nizhni Novgorod State Technical University, Nizhni Novgorod, 603950 Russia \\ ${ }^{f}$ St. Petersburg State Marine Technical University, St. Petersburg, 190008 Russia \\ ${ }^{g}$ EvoLogics, Germany \\ E-mail: bairejinr.ru
}

\begin{abstract}
The first stage of the Baikal-GVD neutrino telescope will be composed of 2304 sensors, Optical Modules (OMs), installed deep underwater in Lake Baikal. In April 2015 the first autonomous part of the future detector, called cluster, was installed. We describe developed calibration methods which use OM LEDs, the calibration LED source, atmospheric muons and discuss the performance of these methods.
\end{abstract}

The 34th International Cosmic Ray Conference,

30 July- 6 August, 2015

The Hague, The Netherlands

\footnotetext{
* Speaker.
} 


\section{Introduction}

The Baikal-GVD project is a logical extension of the research and development work performed on the last several years by the Baikal Collaboration $[1,2,3,4,5,6]$. The detection of highenergy neutrinos has been demonstrated with the NT200 telescope. This achievement presents a proof of concept for commissioning the new instrument, Gigaton Volume Detector (Baikal-GVD), with superior performance and an effective telescope size at or above the kilometer scale. BaikalGVD will be a research infrastructure aimed primarily at studying astrophysical neutrino fluxes and particularly at mapping the high-energy neutrino sky in the Southern Hemisphere including the region of the galactic center.

The apparatus will utilize deep water of Lake Baikal instrumented with light sensors that detect the Cherenkov radiation emitted by the secondary charged particles produced in interactions of high-energy neutrinos inside or near the detector. Reconstruction of location, direction and energy of the secondary particles can be achieved using the time, position and charge information of the hits arriving at the OMs. A good calibration of the detector is necessary in order to achieve an optimal performance.

\section{The first cluster "Dubna"}

The prototyping phase of the Baikal-GVD project has been concluded in April 2015 with the deployment of the first cluster in Lake Baikal. It consists of 192 optical modules (OM) arranged on eight $345 \mathrm{~m}$ long strings (7 peripheral strings located at $40 \mathrm{~m}$ distances from the central one). Each string comprises 24 OMs spaced by $15 \mathrm{~m}$ at depths of 900 to $1250 \mathrm{~m}$ below the surface (see Fig.1). The cluster also comprises an acoustic positioning system, connected to shore by electro-optical cable.

The optical modules are grouped into sections. It is the basic detection units of the array. Each section includes $12 \mathrm{OMs}$ and the central electronic module (CeM). PMT signals from all OMs are transmitted through $90 \mathrm{~m}$ long coaxial cables to the $\mathrm{CeM}$, where they are digitized by custom-made ADC boards with $200 \mathrm{MHz}$ sampling rate. A request of the section trigger is transferred from the Master board to the cluster DAQ center, where a global trigger for all sections is formed. The basic section trigger is hitting two neighbour channels with high (4.0 p.e.) and low ( 0.5 p.e.) thresholds in 90 ns time window [7].

The waveform information in $5 \mu$ s from all measuring channels of the section is transferred to the Master board located in the CeM. The Master board provides data readout from ADC, the section trigger logic, control of the section operation and a connection via local Ethernet to the cluster DAQ center. A request of the section trigger is transferred from the Master board through a string communication module $(\mathrm{CoM})$ to the cluster DAQ-center, where a global trigger for all sections is generated. The global trigger initiates data transmission from all sections to shore.

In order to minimize the data flow, only parts of waveforms around a point that exceeds the 0.2 p.e. level are selected and transferred to shore (Fig.2). This allows to reduce the data flow by factor of 15 .

A special offline algorithm extracts PMT pulses from waveforms and finds theirs characteristics. Pedestals are obtained individually for each pulse, and mean and RMS of pedestal extracted 


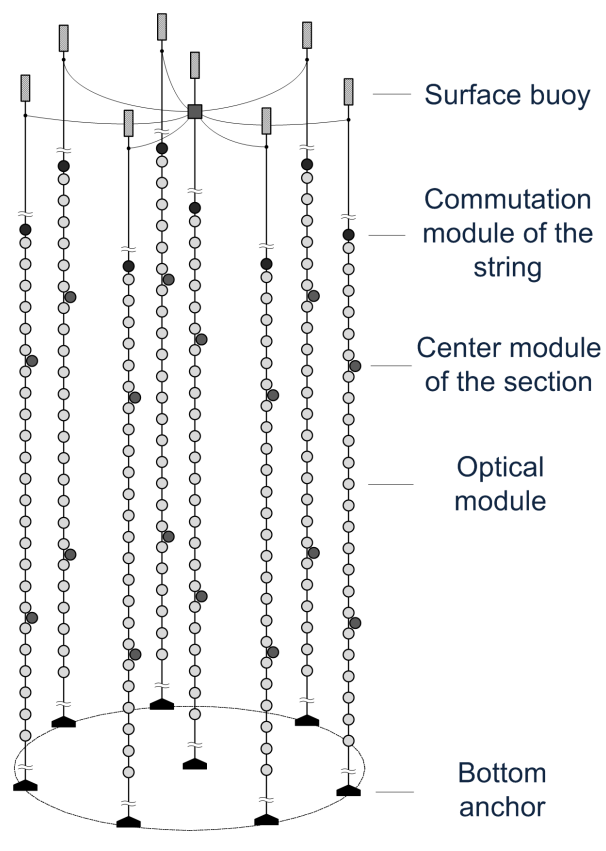

Figure 1: Schematic view of the first Baikal-GVD cluster, consisting of eight $345 \mathrm{~m}$ long strings.

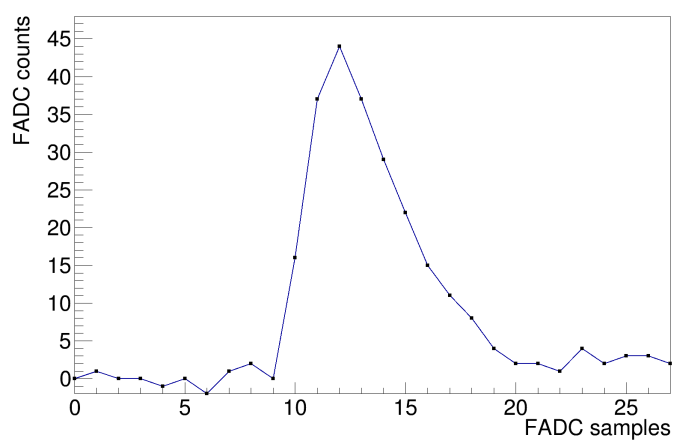

Figure 2: A PMT waveform that is selected by the Master board firmware.

from the first 10 samples of the waveform which are saved specially for this purpose. A PMT pulse is found if there are few points above a level of four RMS of the pedestal. After that amplitude maximum, charge etc. are extracted. Due to optimization a half maximum time of a pulse is taken as the main time mark. The dependence of the time mark of the pulse on its charge was obtained and used as correction for the further analysis [8].

\section{Time calibration}

Precise measurement of the relative photon arrival times at the OMs is crucial for good event reconstruction. The time calibration of the measuring channels aims at control of the relative offsets in times between the PMTs. Such relative offsets for OMs belonging to one section are caused by 


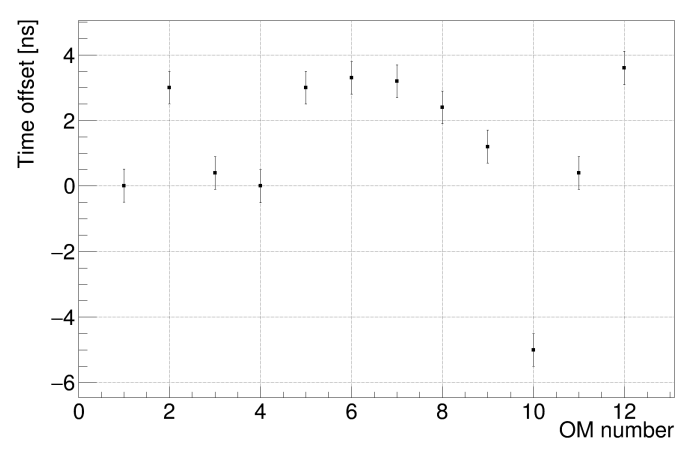

Figure 3: Time offsets for OMs belonging to one section related to the time of the first OM in a section.

internal PMT delay and a delay along the $90 \mathrm{~m}$ long cable connecting OM and CeM. Both delays are measured in the laboratory. Cable delays are the same during array operation. PMT delays are also measured by the following in-situ calibration procedure. There is a reference pulse for this purpose, which is generated by the OM controller right to the measuring channel and gives a time mark of the start time of the OMs LED flash. From the measured difference between times of the LED signal and the reference pulse the PMT delay is obtained.

Figure 3 shows the sum of the obtained PMT and cable delays for OMs belonging to one section related to the first $\mathrm{OM}$ in a section. These time offsets are used as reference time constants that are corrected by the following in-situ calibration procedure.

\subsection{Time calibration with OM LED flashers}

A straightforward test of the obtained time calibration is to pulse the internal OM LEDs (Fig. 4) and measure the arrival time of photons at the own OM and the OMs above. The dominant wavelength of the LEDs is $470 \mathrm{~nm}$, the LED pulse has a width of about $5 \mathrm{~ns}$ (FWHM). There is a possibility of independent regulation of the LED light intensity and delay between flashes of two LEDs. The crosstalk between LED channels is less than $1 \%$.

Because of the distances between neighboured OMs are small enough, effect of light scattering is negligible. Figure 5 shows mean the time differences between hits on OMs subtracting the time of light flight between them and using the obtained time offsets. Because of some time differences are not zero, systematics errors are observed and are used for time offset correction. They were found better than $2 \mathrm{~ns}$ for most of the OMs, confirming the precision of time synchronization.

Internal OM LEDs are also used for time calibration between sections which are located on the same string. For this purpose the LED in the highest OM in the lower section flashes the lowest $\mathrm{OM}$ in the higher section. In that way the time offset between two sections as a whole was found.

\subsection{Time calibration with LED Beacon}

The OM LEDs are faced vertically hence cannot be used for interstring time calibration. For this purpose a LED Beacon was installed. The LED Beacon contains a flasher board which holds 12 LEDs. Six of them point upward and six point horizontally. The LEDs flash light individually with programmable amplitude and rate. 


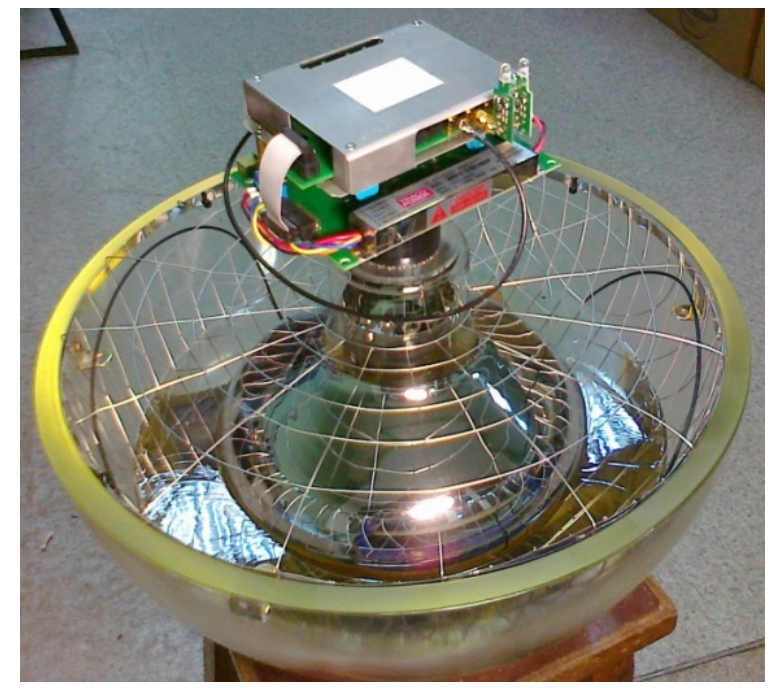

Figure 4: Picture of the Baikal-GVD Optical Module. Two LEDs are seen at the top of the OM electronics.

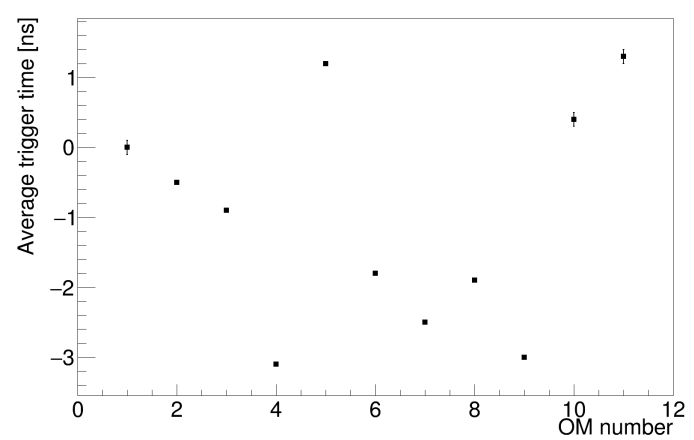

Figure 5: Mean time offsets for OMs belonging to one section related to time offset of the first OM in a section. Error bars are errors of mean.

The LED Beacon is located on one peripheral string between 6th and 7th OMs of the upper section. It flashes OMs on the same horizon in all other strings and thereby provides interstring time calibration, together with the information from the acoustic positioning system.

\subsection{Time calibration with muons}

The relative time offset between two measuring channels can also be obtained via the time difference of signals from down-going cosmic-ray muons on neighboring OMs. In this case, pairwise channel delays are equal to the distance between experimental and modeled muon time difference distributions. Also, comparing muon time differences for two sets of experimental data provides insight into the stability of channel delays and channel parameters in general.

Separating a muon signal from background noise is a two-step process. First, event impulses are filtered by a causality noise reduction algorithm. Each channel, that has at least one non-filtered impulse, is considered a "hit channel". If a hit channel has two impulses or more, only the first 


$\begin{array}{lrr} & \text { Impulse labeled as signal } & \text { Impulse labeled as noise } \\ \text { Signal } & 30.3 \% & 69.7 \% \\ \text { Noise } & 1.1 \% & 98.9 \%\end{array}$

Table 1: Muon signal classifier performance for modeling 2014

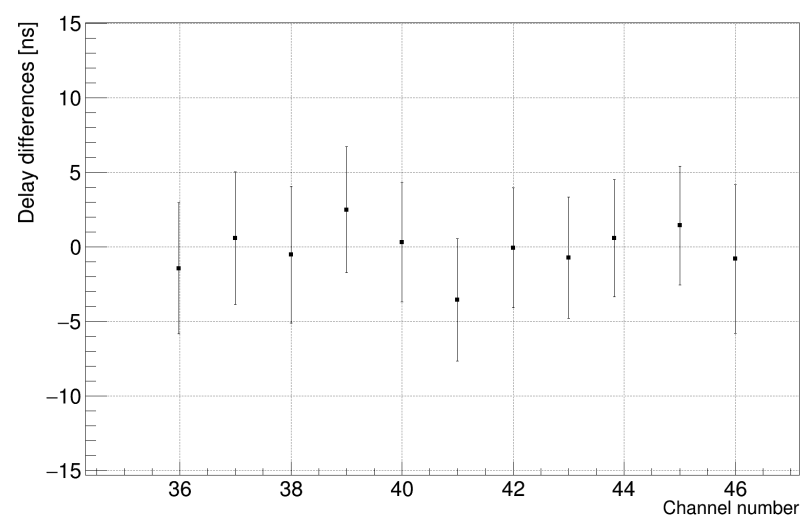

Figure 6: Pairwise channel delay difference for May and June 2015

one is used for the following analysis. The next step is determining which channels have a muon signal and can, consequently, be used for muon calibration. A hit channel is assumed to have a muon signal if either both of its neighboring channels are hit, or at least one of them has a muon signal. As an additional requirement, at least one in three consecutive channels with muon signals is required to have an impulse that exceeds a charge threshold. This approach yields a conservative impulse classifier, as shown on Table 1.

With muon impulses retrieved from the data, muon time-difference distributions can be obtained for pairs of neighboring channels. If this process is repeated for two different sets of events, for each channel pair there will be two time-difference distributions, one for each set. The computed distance between these distributions yields change in pairwise channel delay from one set to another. The distance is evaluated by picking a similarity function $M\left(\Delta T_{1}, \Delta T_{2}(\tau)\right)$ over $\tau$, where $\Delta T_{1}$ is the time difference distribution for the first set and $\Delta T_{2}(\tau)$ is the time difference distribution for the second set shifted by $\tau$. The value of $\tau$, that maximizes $M$, is considered the distance between $\Delta T_{1}$ and $\Delta T_{2}$. Currently, $M$ is defined as the integral of intersection of the time-difference distributions.

If for a pair of channels one muon time-difference distribution is obtained from data and another by modeling, then $\tau$ equals pairwise channel delay and can be used for time calibration. If the time-difference distributions are from different runs, then $\tau$ equals the difference between their corresponding pairwise channel delays. The latter approach has been used to determine the stability of channel delays. The difference between pairwise channel delays for data taken in May and June 2015 (as shown in Fig. 6) is zero within errors, which indicates stability of pairwise channel delays and, therefore, time calibration.

At this time, the muon calibration error is approximately 4-5 ns. It can be lowered by utilizing 


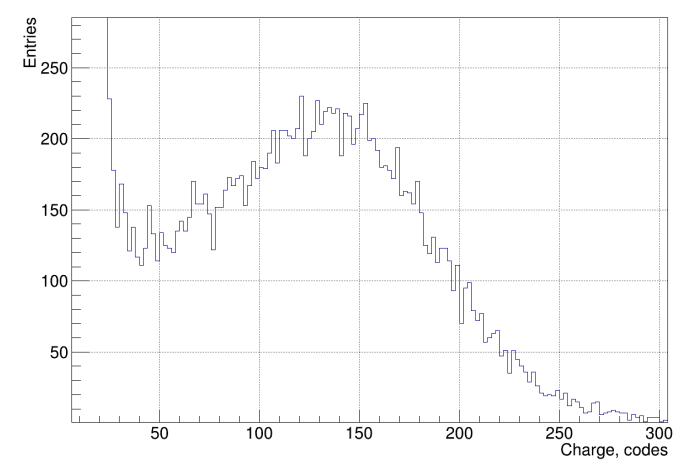

Figure 7: One photoelectron distribution obtained from noise

a more advanced muon calibration technique that uses a muon track reconstruction procedure.

\section{Amplitude calibration}

The purpose of the amplitude calibration is to translate the pulse charge into the number of photoelectrons. A standard procedure based on the position of the single photoelectron distribution (s.p.e) has been applied. The pulses of two OM LEDs are used. The intensity of the first LED is fitted to provide a detection of s.p.e signals with a detection probability of about $10 \%$. These pulses are used to measure the s.p.e distribution of channel signals. Pulses of the second LED with intensities corresponding to about 50 p.e. are delayed for $500 \mathrm{~ns}$ and are used as a trigger.

The optical activity from chemilumenescence of deep water in Lake Baikal produces single photons. An alternate procedure of charge calibration uses these noise events to study the single photoelectron peak (fig. 7).

\section{Conclusions}

The methods and systems used to perform the time and amplitude calibration for the BaikalGVD neutrino detector have been reviewed. They have been successfully tested in situ by experimental data. Cross-checks between two independent time calibration methods give an agreement of 2 ns for most of the OMs.

\section{Acknowledgments}

This work was supported by the Russian Foundation for Basic Research (grants 13-02-12221, 14-02-00972).

\section{References}

[1] A. Avrorin et al., The prototyping/early construction phase of the BAIKAL-GVD project, NIM A742 82. 
[2] V. Aynutdinov et al., The prototype string for the km3-scale Baikal neutrino telescope, NIM A602 227.

[3] V. Aynutdinov et al., The BAIKAL neutrino experiment, NIM A626 13.

[4] V. Aynutdinov et al., The gigaton volume detector in Lake Baikal, NIM A639 30.

[5] A. Avrorin et al., Current status of the Baikal-GVD project, NIM A725 23.

[6] A. Avrorin et al., Status and recent results of the Baikal-GVD project, Phys. of Part. and Nucl. 46211.

[7] A. Avrorin et al., Data acquisition system of the NT1000 Baikal neutrino telescope, Instr. and Exp. Tech. 57262.

[8] A.D.Avrorin et al., The optical module of the Baikal-GVD neutrino telescope, this proceedings

[9] A. Avrorin et al., The first construction phase of the Baikal-GVD neutrino telescope, this proceedings. 